\title{
Analysis of Degradation Mechanisms and Durability Assessment of Graphene-based MPLs for PEM Fuel Cells
}

\author{
S. Latorrata, P. Gallo Stampino, L. Scandola, C. Cristiani and G. Dotelli \\ Department of Chemistry, Materials and Chemical Engineering, Politecnico di Milano, \\ piazza Leonardo da Vinci, 32, 20133 Milan, Italy
}

\begin{abstract}
Novel graphene-based microporous layers (MPLs) have been developed as a possible alternative to conventional carbon blackbased MPLs aiming to enhance electrical performance and durability of PEM fuel cell systems. Graphene nanoplatelets (GNPs) have been used as carbonaceous conductive phase in the ink formulation for MPL due to their low electrical resistance, high surface area and mechanical stability. The novel MPLs exhibited more homogeneous and less cracked surfaces compared to MPLs containing carbon black. Such enhanced MPL surface resulted in a better electrical contact between MPL and fuel cell electrode and a consequent lower ohmic resistance. Moreover, chemical and mechanical accelerated stress tests were developed in order to evaluate durability of the prepared samples and the most detrimental degradation mechanism. Fresh MPLs showed very similar electrochemical behaviors in running fuel cells while GNPs-based MPLs proved to be more resistant against both stress factors than conventional ones.
\end{abstract}

\section{Introduction}

Water management in polymer electrolyte membrane fuel cells (PEMFCs) is a crucial aspect which must be properly addressed in order to have high and constant efficiency during device operation. In this respect, gas diffusion medium (GDM) is a fundamental component for a PEMFC because it is inserted between the flow field and the catalytic layer aiming to guarantee a correct balance between the inlet (with humidified gases) and generated water (by the oxygen reduction reaction). Indeed, an excessive accumulation of water could rise diffusive limitations and lead to flooding, above all at high current densities values, where reaction rate in higher. Therefore GDM needs to be made hydrophobic and usually such target is accomplished by using polytetrafluoroethylene (PTFE) or some derivatives of it (1-5). GDM is formed by a carbon cloth or paper macroporous substrate (gas diffusion layer, GDL) and a micro-porous layer (MPL) made from an ink and deposited directly onto GDL. Such a coating improves the smoothness of the GDL surface allowing a better contact with the catalytic layer thus reducing overall cell ohmic resistance (1). MPLs are mainly prepared from inks containing carbon conductive particles (carbon black, nanotubes or graphene) and PTFE, the latter used as a hydrophobic agent. For some years now, our research group has demonstrated the effectiveness of replacing PTFE with fluorinated ethylene propylene (FEP) in order to improve hydrophobic properties of MPLs and, consequently, the water removal $(6,7)$. 
In this work, the carbonaceous conductive phase was changed: graphene nanoplatelets (GNPs) and carbon black (CB) were used to prepare different inks in order to compare the electrochemical performance of the resulting MPLs. GNPs were thought to be beneficial for the enhancement of mass transport and removal of the generated liquid water. GNPs are nanocarbonaceous materials with a mean surface area of $350 \mathrm{~m}^{2} / \mathrm{g}$ and functional groups, like carbonyl and carboxyl, at the edges of the platelets. The resistivity of GNPs as a function of applied pressure shows values from 0.59 to $0.11 \Omega \mathrm{cm}$. A typical carbon black powder used to prepare MPLs, such as Vulcan XC72, has a mean surface area of $262 \mathrm{~m}^{2} / \mathrm{g}$; furthermore, its electrical resistivity varies from 0.67 to $0.10 \Omega \mathrm{cm}$. So it could be useful to replace Vulcan XC72 with GNPs in MPL design; a higher surface area and a better resistivity than carbon powder should guarantee higher performances in both mass transport and generated power.

\section{Experimental}

Carbon clothes GDLs (S5, supplied by the Italian company SAATI) were pre-treated to make them suitable for the subsequent MPL coating. Indeed, a hydrophobic surface is also needed for the backing cloth for avoiding liquid loss (i.e., ink liquid components passing through GDL itself) during the coating process. GDLs were soaked in a $12 \%$ by weight FEP solution (7); two different inks containing carbonaceous phase, e.g. GNP or $\mathrm{CB}$, mixed with FEP, water and isopropyl alcohol (IPA), with composition reported in Table I, were deposited onto GDLs by blade-coating technique and then they were thermal treated $\left(260{ }^{\circ} \mathrm{C}\right.$ for 30 minutes) thus producing the MPL.

TABLE I. Composition of the inks used to prepare MPLs.

\begin{tabular}{cccccc}
\hline Sample & CB $(\mathbf{g})$ & GNP $(\mathbf{g})$ & FEP $(\mathbf{g})$ & Water $(\mathbf{g})$ & IPA $(\mathbf{g})$ \\
\hline ink-CB & 4 & - & 0.48 & 30.4 & 0.71 \\
ink-GNP & - & 4 & 0.48 & 30.4 & 0.71 \\
\hline
\end{tabular}

Electrochemical tests were performed in a single cell with a commercial catalyst coated membrane (Baltic Fuel Cells, Germany) consisting of Nafion 212 and Pt/C electrodes, with a platinum loading of $0.2 \mathrm{mg} / \mathrm{cm}^{2}$ at the anode and $0.4 \mathrm{mg} / \mathrm{cm}^{2}$ at the cathode, and an active area of $23 \mathrm{~cm}^{2}$. The cell testing was run at $60{ }^{\circ} \mathrm{C}$ with different relative humidities (RH $100 \%$ and $60 \%$ ). The compression of GDMs was fixed at $30 \%$ original thickness and kept constant with the use of uncompressible glass fiber gaskets. In a previous work, such value of compression has been demonstrated to be optimal for improving cell performance, especially at high current density (8). Electrochemical Impedance Spectroscopy (EIS) was carried out using a Frequency Response Analyzer, FRA (Solartron Group), in the frequency range from $0.5 \mathrm{~Hz}$ to $10.0 \mathrm{kHz}$ (9). The typical spectrum of an operating fuel cell is composed of one, two or, more rarely, three arcs, whose origin can be ascribed to activation polarization and concentration polarization (7, 10, 11). The ZView software (Scribner Associates) was employed to fit experimental data by means of equivalent circuits described in an authors' previous work (7) in order to get ohmic, charge transfer and mass transfer resistances.

Durability is still a critical issue to be faced in fuel cells field in order to have a real competition with conventional energy generators. While for membranes, catalysts and 
bipolar plates many standard protocols for testing their durability through accelerated stress tests (ASTs) have been designed, this is not the case for GDLs and MPLs (7, 1216). Thus, ad-hoc chemical and mechanical ASTs were developed in this work. Two exsitu ASTs were developed to analyze the effect of both mechanical and chemical degradation phenomena of GDMs on fuel cell performance. The chemical AST consisted in soaking GDMs in a $20 \%$ by volume sulfuric acid solution at a $\mathrm{pH}$ value which was far below the typical environment PEM fuel cell $\mathrm{pH}$ (2-3), for a total time of $1000 \mathrm{~h}$ (7). This condition was expected to accelerate the chemical degradation of GDMs. The mechanical AST was based on a literature study (13), but a simpler system was set up and already adopted in Reference (7). A dummy cell was assembled with two GDMs at anodic and cathodic side, separated by an inert PTFE foil, to avoid any possible chemical or electrochemical stress. Only air was supplied to either side (7) with twofold flow rates with respect to those employed during standard running $(0.25$ and $1.0 \mathrm{NL} / \mathrm{min}$ for hydrogen and air, respectively) for making mechanical degradation faster. Air was fed for $1000 \mathrm{~h}$ continuously, therefore multiple compression/decompression cycles were avoided. The purpose was to test GDMs mechanical resistance and endurance of MPLs, namely the ability of MPLs to avoid detachment from the GDL substrate, and to relate ASTs effect to fuel cell performance.

Different characterizations were carried out on GDMs before and after electrochemical and stress tests. Scanning electron microscope (Cambridge Stereoscan 360) was used for the morphological analyses of surface of the GDMs. Samples were previously gold coated to prevent charging effects. The samples hydrophobicity was assessed by static contact angles technique; the measurements were performed, on the MPL side, according to the sessile drop technique using an OCA 20 instrument (Dataphysics, Germany). Values reported in this work are the result of the average of ten measurements (7).

\section{Results and Discussion}

Figure 1 shows polarization and power density curves of fuel cells assembled with standard GDMs based on carbon black compared with those obtained with novel GNPbased samples and working at different operating conditions, as described in the Experimental section. 

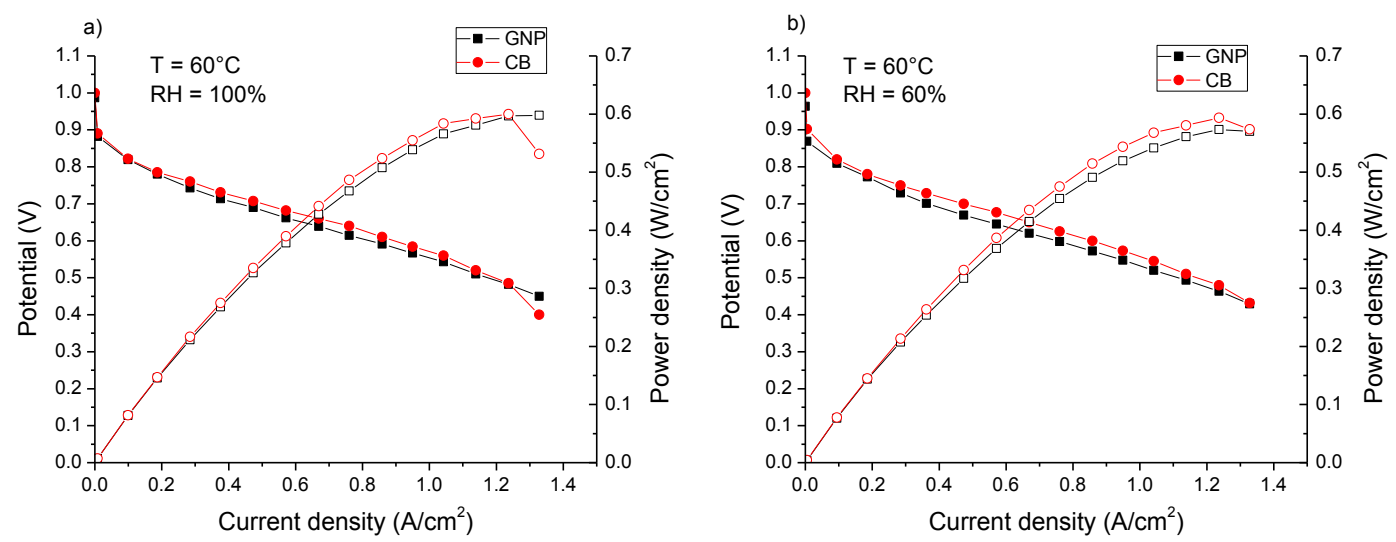

Figure 1. Polarization and power density curves obtained at $60{ }^{\circ} \mathrm{C}$ and $\mathrm{RH} 100 \%$ (a) and RH $60 \%$ (b) for running fuel cells assembled with CB- and GNP-based MPLs.

Electrical performances of the samples are very similar in both $\mathrm{RH}$ conditions with maximum power density close to $0.6 \mathrm{~W} / \mathrm{cm}^{2}$. Overall, CB-based GDMs show slightly higher performance even though no significant difference can be identified. Potential values, which are directly proportional to global cell efficiency (7), are very similar to those measured for GNPs-containing sample over the whole range of generated current. However, a small difference can be highlighted at high humidity, i.e. RH $100 \%$ (Figure 1a): GDMs containing CB exhibit a higher voltage loss than GNPs-based samples at very high current density denoting a higher concentration polarization. This might be due to a slightly worse water management which might be related to a different MPL surface morphology and it should not depend on differences in wettability. Indeed similar contact angle values were found: GNPs-based GDM showed a value of $152^{\circ} \pm 2^{\circ}$ while a value of $157^{\circ} \pm 3^{\circ}$ was obtained for conventional GDM. Anyway, both of them lie in the superhydrophobic region therefore being able to efficiently remove excess water from the device.

Figure 2 shows the difference between the surface of conventional and GNPs-based MPLs. It is clear that the presence of GNPs allowed to reduce sharply the number of cracks and their size; cracks seem to be instead unavoidable for CB-based MPLs. Such different surface morphology can be ascribed to the effect of the graphene platelets which are more likely to coalesce compared to carbon black nanoparticles and to avoid the "mudcrack-like" shape as for conventional MPLs. The more compact surface of GNPbased samples can also play an important role in decreasing the overall ohmic resistance of the operating fuel cell since a better electrical contact between MPL and catalyst layer can be accomplished due to the absence of cracks. Moreover, it is thought that it might also improve adhesion between MPL and the GDL substrate as well as influence the mass transport properties since cracks can gather more water molecules, coming both from humidification of reactant gases and from redox reaction, which may increase diffusion resistances by hindering the passage of reagents towards electrode active sites. 

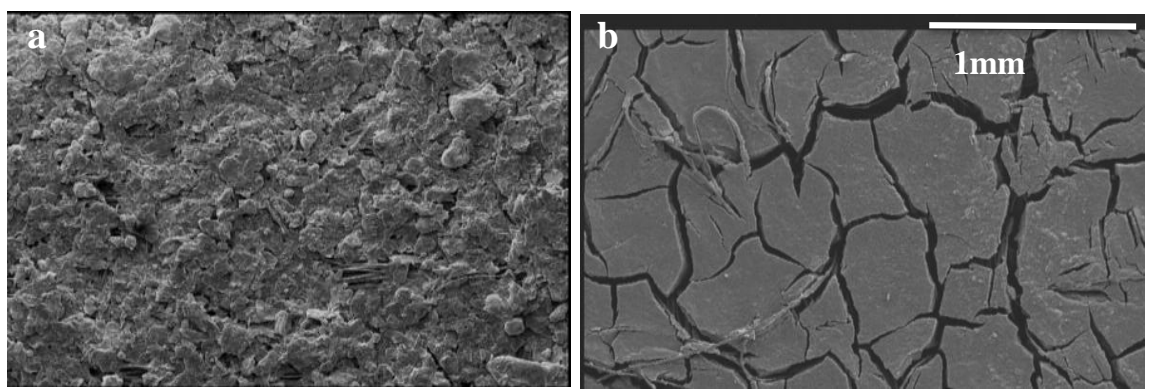

Figure 2. SEM images of GNPs- (a) and CB-based (b) MPLs.

As a matter of fact, trend of ohmic resistance and mass transfer resistance as a function of current density for both samples at any operating conditions are reported in Figure 3.
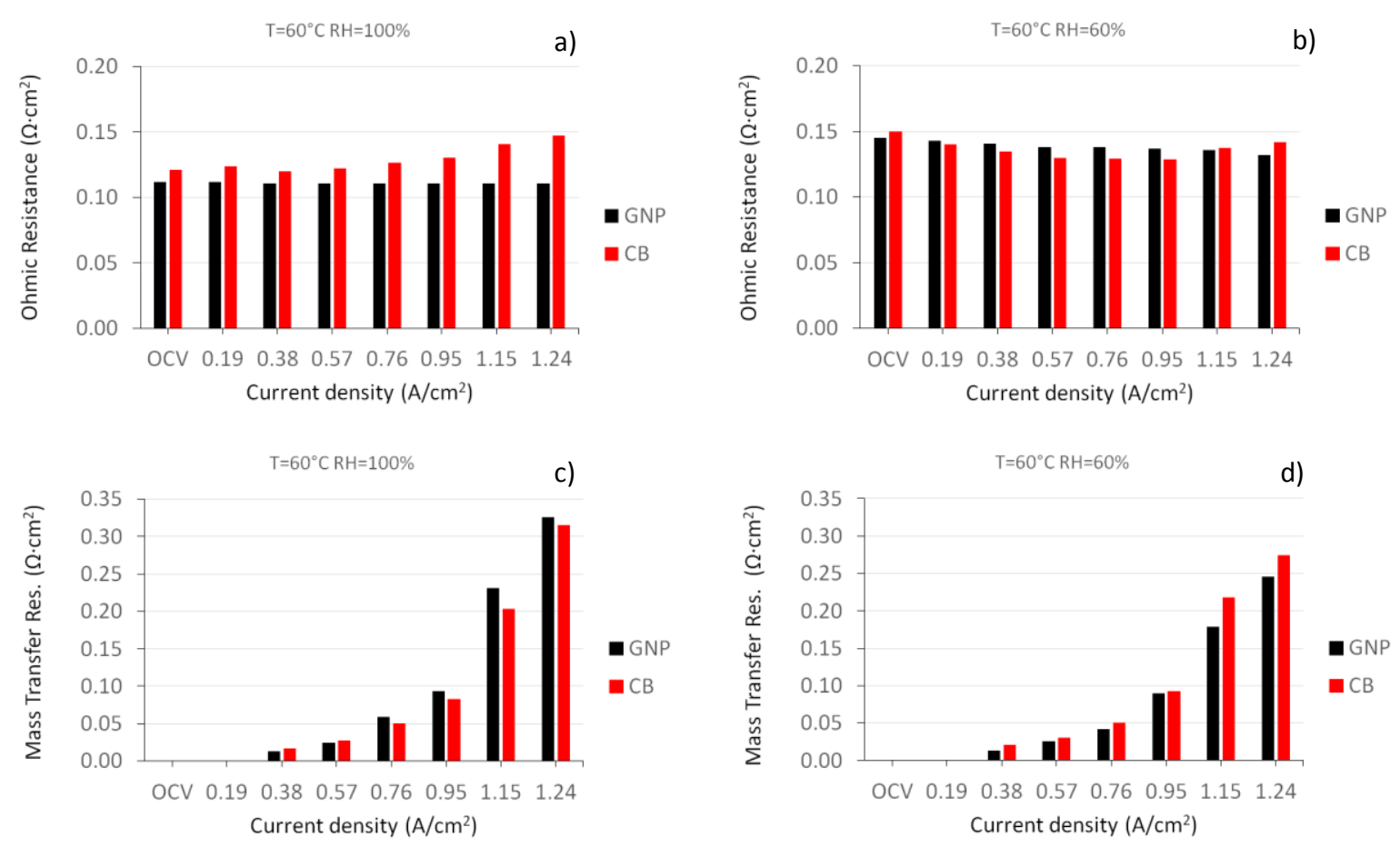

Figure 3. Trend of ohmic (a, b) and mass transfer resistance (c, d) as a function of current density for GNPs- and CB-based samples. Operating conditions: $60{ }^{\circ} \mathrm{C}$ and $\mathrm{RH} 100 \%$ (a, c) and $60{ }^{\circ} \mathrm{C}$ and $\mathrm{RH} 60 \%(\mathrm{~b}, \mathrm{~d})$.

GNPs allowed to reduce ohmic resistance at RH $100 \%$ while very small differences can be noticed at RH $60 \%$. Such behavior might be due to a combination of a slightly higher wettability of the GDM which can increase the hydration of the electrolyte, especially at high humidity, and better electrical contact between the MPL and the catalyst layer of the MEA. As far as mass transfer resistance is concerned, it can be observed a general expected trend for both GDMs: such resistance increases upon increasing current density because of a faster and higher production of water. GNPsbased GDMs are able in reducing diffusive limitation, even though lightly, at RH $60 \%$. 
Figure 4 and Figure 5 show, respectively, polarization (and related power density) curves and trend of ohmic resistance and mass transfer resistance as a function of current density upon ASTs.
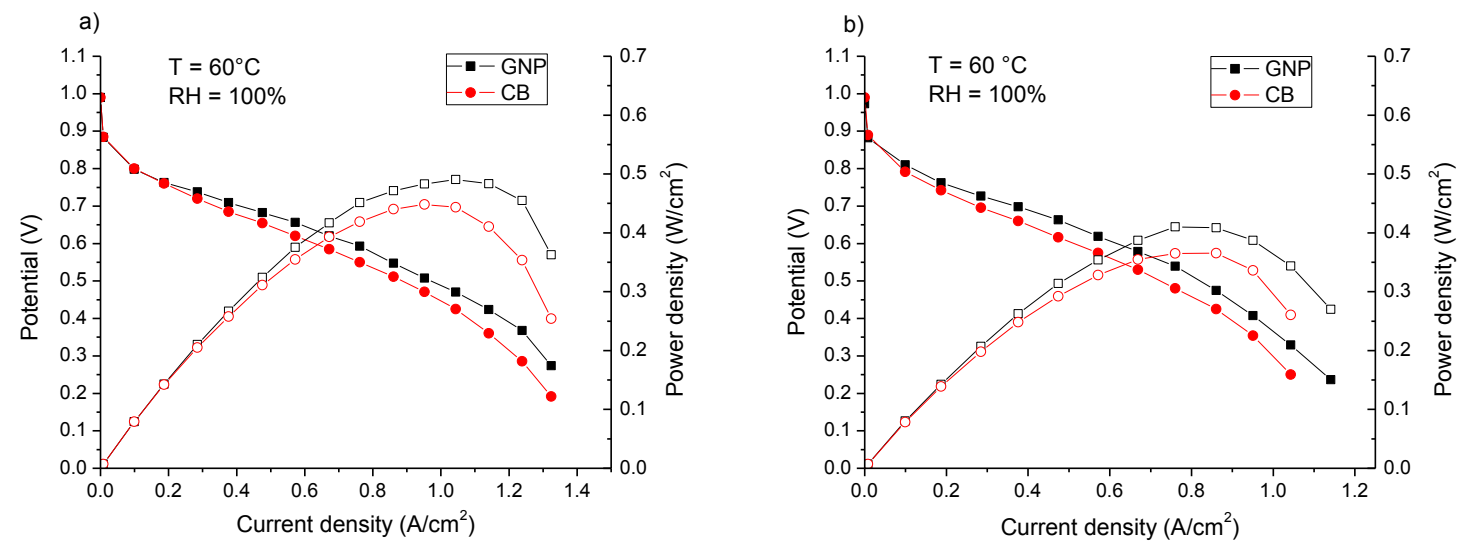

Figure 4. Polarization and power density curves obtained for chemically (a) and mechanically (b) stressed GDMs. Operating conditions: $60{ }^{\circ} \mathrm{C}$ and RH $100 \%$.

An obvious and expected pronounced worsening of the performance compared to fresh (i.e. not damaged by ASTs) samples can be observed. It is worth noting that novel GNPs-based GDMs allowed to obtain higher performances after both mechanical and chemical AST likely due to a better adhesion of the MPL deposited onto the GDL. As previously reported (7), mechanical degradation seems to be more detrimental than chemical for both samples. Such finding can be confirmed by analyzing results of Figure 5. Indeed, higher ohmic and mass transfer resistances have been found for mechanically damaged samples. GNPs-based GDMs show a better situation compared to conventional samples also in this case. This result justifies the higher slope in the ohmic region, i.e. the linear part of the polarization curve, as well as the more pronounced concentration losses at high current density values. Such a better behavior for the novel GDMs can be ascribed to less cracked surface which may be able to improve adhesion between components, e.g. MPL and GDL. 

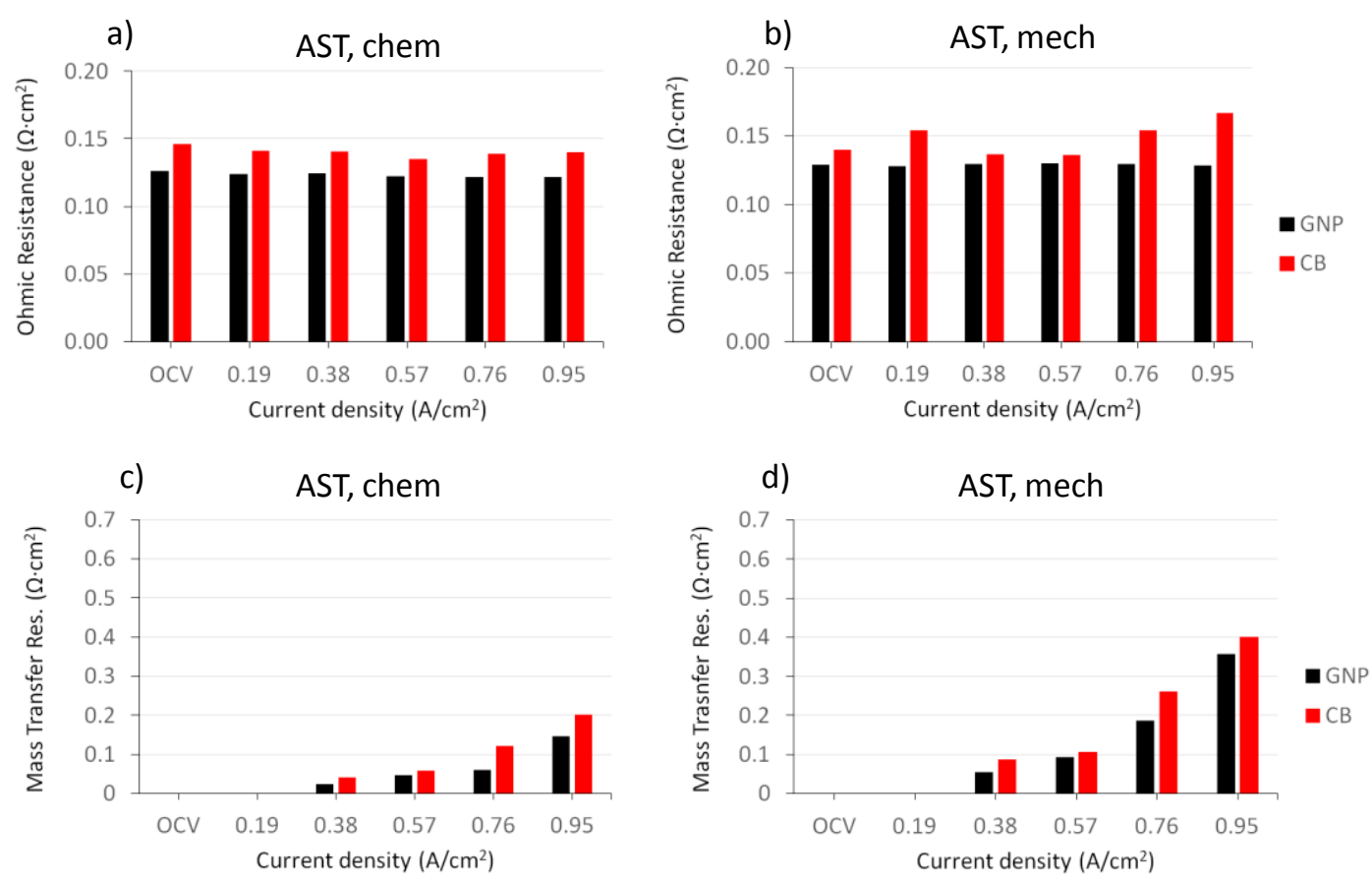

Figure 5. Trend of ohmic $(a, b)$ and mass transfer resistance $(c, d)$ as a function of current density for GNPs- and CB-based samples after chemical $(a, c)$ and mechanical (b, d) AST.

Table II can help summarizing results of the characterization upon ASTs.

TABLE II. Main parameters, i.e. contact angle, change in maximum fuel cell power density, average ohmic resistance and maximum mass transfer resistance, obtained before and after chemical and mechanical ASTs (B-AST: before AST, A-ASTc: after chemical AST, A-ASTm: after mechanical AST).

\begin{tabular}{|c|c|c|c|c|c|c|c|c|c|}
\hline \multirow{2}{*}{ Sample } & \multirow{2}{*}{$\begin{array}{l}\mathrm{CA}\left[{ }^{\circ}\right] \\
\text { B-AST } \\
\end{array}$} & \multicolumn{2}{|c|}{$\mathrm{CA}\left[^{\circ}\right]$} & \multicolumn{2}{|c|}{$\Delta \mathbf{P}_{\mathrm{MAX}}[\%]$} & \multicolumn{2}{|c|}{$\mathbf{R}_{\mathrm{Ohm}}$ av $\left[\Omega \mathrm{cm}^{2}\right]$} & \multicolumn{2}{|c|}{$\mathbf{R}_{\mathrm{mt}} \max \left[\Omega \mathrm{cm}^{2}\right]$} \\
\hline & & A-ASTe & A-ASTm & ASTc & ASTm & ASTc & ASTm & ASTc & ASTm \\
\hline CB & $157 \pm 3$ & $153 \pm 4$ & $145 \pm 3$ & 25.3 & 39.1 & 0.1451 & 0.1479 & 0.2011 & 0.4002 \\
\hline GNP & $152 \pm 2$ & $148 \pm 4$ & $146 \pm 2$ & 17.9 & 31.4 & 0.1232 & 0.1292 & 0.1456 & 0.3564 \\
\hline
\end{tabular}

A very limited decrease of hydrophobicity can be observed for both GDMs; indeed reduced static contact angle (CA) values have been obtained after ASTs but they are still close to the superhydrophobicity limit, i.e. $150^{\circ}$. Therefore, the worsening in electrochemical performance might be more likely due to a change in morphology and adhesion properties of the MPLs surface. By observing the change in maximum power density $\left(\Delta \mathrm{P}_{\max }\right)$ and the values of average ohmic resistances and maximum mass transfer resistances after ASTs, the benefits introduced by the use of GNPs as conductive phase in the ink for MPLs preparation should be clear once again: novel MPLs have been able to reduce such change and to keep electrochemical parameters lower than those exhibited by CB-based MPLs. 


\section{Conclusions}

Graphene nanoplatelets (GNPs) were used in the preparation of conductive inks to be used for microporous layers (MPLs) deposition onto gas diffusion layers for PEM fuel cells as a possible alternative to conventional carbon black. The aim of such experiments was to improve both electrical performance (in terms of output power density and electrochemical parameters such as ohmic and mass transport resistances) of the whole fuel cell device. GNPs allowed to reduce dramatically cracks of MPLs surface and this positively influenced the durability and the resistance against mechanical and chemical degradation. Indeed accelerated stress tests (ASTs) were developed in order to evaluate the capability of the prepared samples to withstand both chemical and mechanical degradation and novel GNPs-based MPLs showed a better electrical behavior upon such tests. As a matter of fact, they exhibited lower ohmic and diffusive resistances compared to carbon black-based MPLs and a restrained change in maximum output power density. Therefore, despite these preliminary results need a confirmation at different operating conditions and possibly with different starting inks composition, it is thought that GNPs can be used as a valuable alternative to carbon black to enhance both electrical performance and durability of MPLs for PEM fuel cells.

\section{References}

1. S. Park, J. W. Lee and B. N. Popov, Int. J. Hydrogen Energ., 37, 5850 (2012).

2. M. B. Ji and Z. D. Wei, Energies, 2, 1057 (2009).

3. L. Carrette, K. A. Friedrich and U. Stimming, Fuel Cells, 1, 5 (2001).

4. Y. Wang, K. S. Chen, J. Mishler, S. C. Cho and X. C. Adroher, Appl. Energ., 88, 981 (2011).

5. C. Wang, S. B. Wang, L. F. Peng, J. L. Zhang, Z. G. Shao, J. Huang, C. W. Sun, M. G. Ouyang and X. M. He, Energies, 9 (2016).

6. S. Latorrata, R. Balzarotti, P. G. Stampino, C. Cristiani, G. Dotelli and M. Guilizzoni, Prog. Org. Coat., 78, 517 (2015).

7. S. Latorrata, P. G. Stampino, C. Cristiani and G. Dotelli, Int. J. Hydrogen Energ., 40, 14596 (2015).

8. G. Dotelli, L. Omati, P. G. Stampino, P. Grassini and D. Brivio, J. Power Sources, 196, 8955 (2011).

9. P. G. Stampino, S. Latorrata, D. Molina, S. Turri, M. Levi and G. Dotelli, Solid State Ionics, 216, 100 (2012).

10. R. B. Ferreira, D. S. Falcao, V. B. Oliveira and A. M. F. R. Pinto, Electrochim. Acta, 224, 337 (2017).

11. M. Schulze, N. Wagner, T. Kaz and K. A. Friedrich, Electrochim. Acta, 52, 2328 (2007).

12. X. Z. Yuan, H. Li, S. S. Zhang, J. Martin and H. J. Wang, J. Power Sources, 196, 9107 (2011).

13. J. H. Chun, D. H. Jo, S. G. Kim, S. H. Park, C. H. Lee and S. H. Kim, Renew. Energ., 48, 35 (2012).

14. N. Yousfi-Steiner, P. Mocoteguy, D. Candusso and D. Hissel, J. Power Sources, 194, 130 (2009).

15. B. B. Wu, M. Zhao, W. Y. Shi, W. M. Liu, J. G. Liu, D. M. Xing, Y. F. Yao, Z. J. Hou, P. W. Ming, J. Gu and Z. G. Zou, Int. J. Hydrogen Energ., 39, 14381 (2014). 
16. S. S. Zhang, X. Z. Yuan, H. J. Wang, W. Merida, H. Zhu, J. Shen, S. H. Wu and J. J. Zhang, Int. J. Hydrogen Energ., 34, 388 (2009). 